ISSN 1409-2441

www.ucr.ac.cr
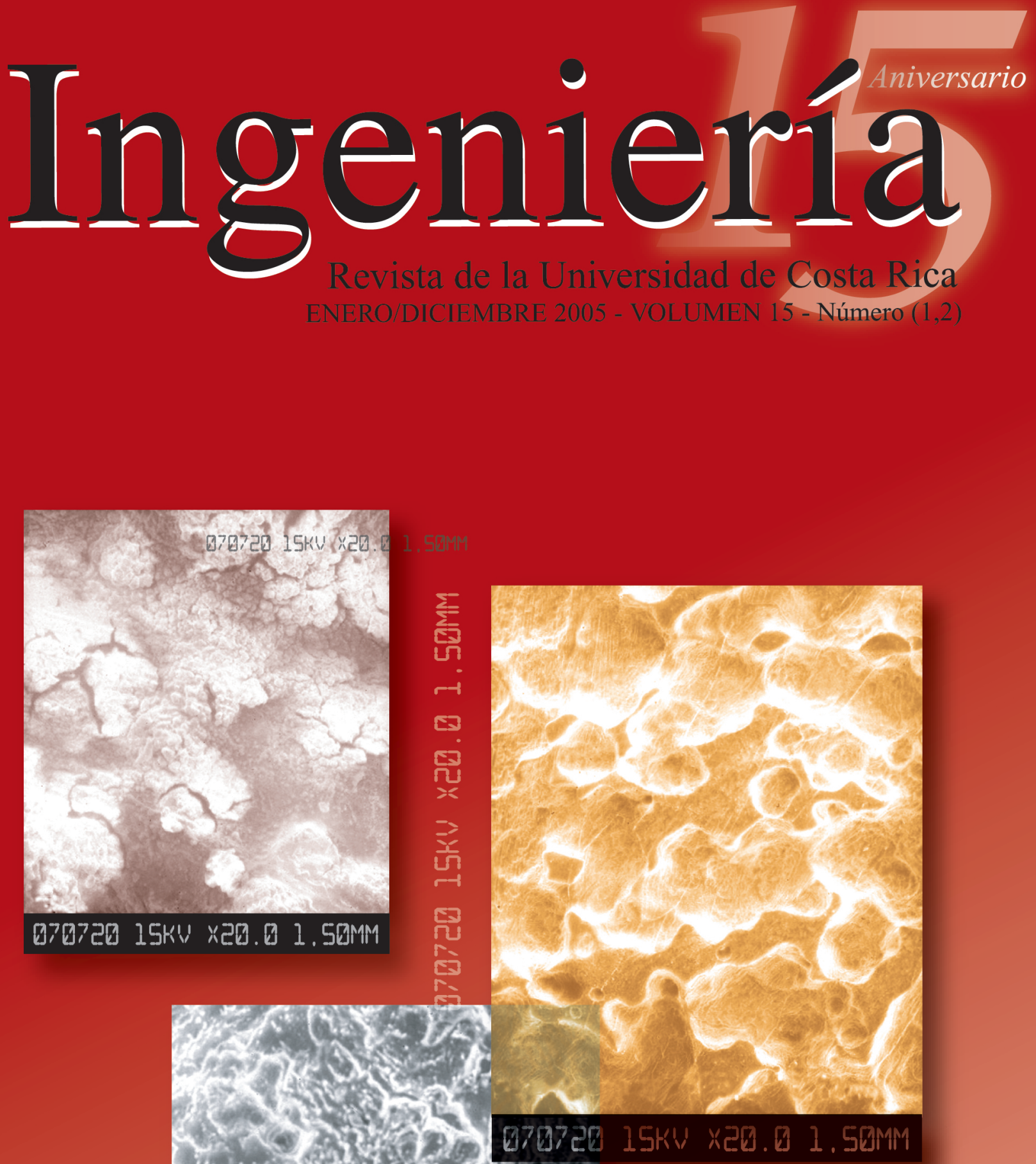

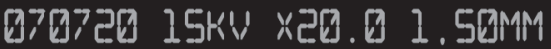

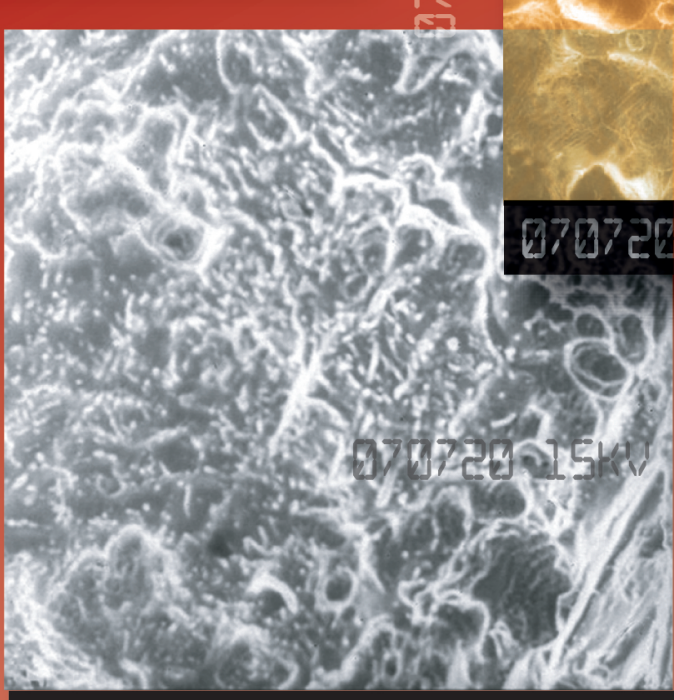

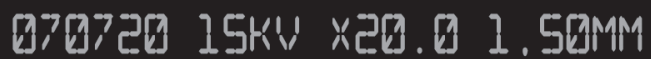


Nota

Técnica 



\title{
SELLOS Y TRATAMIENTOS CONTRA FUEGO
}

\author{
Jhymer Rojas Vásquez
}

\begin{abstract}
Resumen
Los sellos contra fuego son construcciones específicas que constan de ensambles de pared o piso, de uno o varios elementos penetrantes que pasan a través de una abertura en dicho ensamble, además de los materiales diseñados para impedir la expansión del fuego a través de las aberturas.
\end{abstract}

El término tratamientos contra fuego se utiliza, principalmente, en los recubrimientos utilizados para tratar materiales combustibles de construcción.

Palabras clave: propagación, retardantes, barreras, cortafuegos, sellantes, intumescencia, ASTM-E814

Abstract

Fire seals are a wall or floor assembly through witch one or more penetrating elements pass (such as piping or wiring) along with any necessary materials to keep fire or smoke from propagating through the opening.

The term "fire treated" mainly addresses coverings used to treat combustible construction materials.

Key words: spread, retardant, barriers, firestops, sealant, intumescent, ASTM-E814

Recibido: 22 de junio del 2005 / Aprobado: 22 de julio del 2005

\section{CLASIFICACIÓN DE LOS SELLOS CONTRA FUEGO}

Los sellos contra fuego deben ser no combustibles y con un punto de fusión que les permita permanecer inalterados durante su exposición al fuego. Las placas de yeso, chapas metálicas, ladrillo, cementos, fibras minerales y planchas de fibras cerámicas, son algunos ejemplos de materiales utilizados normalmente para contener el fuego.

Los fabricantes de sellos contra fuego deben estar listados en un Directorio de Resistencia al Fuego, suscrito por una organización reconocida como UL (Underwriters Laboratories). Los ensambles y los dispositivos son, los probados y listados. En el cuadro 1 se muestra la simbología utilizada por UL.

La norma ASTM E-814 se creó para evaluar los cortafuego que se instalan paraevitar la propagación del fuego a través de aberturas. Esta norma se publicó por primera vez en 1981. El ensayo que propone la ASTM E-814 es muy parecido al de la ASTM E-119, pero se realiza a mucha menor escala y a una presión positiva determinada. Además, todos los elementos del ensayo están sujetos a un ensayo de manguera similar al exigido para ciertos tipos de construcción, que se prueban según la norma ASTM E-119. Esta norma requiere probar, por lo menos, una muestra de $9 \mathrm{~m}^{2}$ de pared y otra de $16,2 \mathrm{~m}^{2}$ de suelo. En la ASTM E-814 se puede probar una muestra de pared o de suelo de menos de $0,81 \mathrm{~m}^{2}$. En esta última norma, el horno se calienta siguiendo el esquema de temperaturas de la ASTM E-119.

Los sellos cortafuego se clasifican en dos categorías, según la duración de su protección: $\mathrm{F}$ y T. La clasificación F ('F rating') es el tiempo en horas o fracciones en que el sistema cortafuegos conserva su integridad física, aunque esté expuesto al fuego y a los chorros de las mangueras, y evita 
que pase la llama a la parte no expuesta al incendio. La clasificación T ('T rating') es el tiempo en horas que tarda el lado del sello no expuesto al fuego en incrementar $163{ }^{\circ} \mathrm{C}\left(325^{\circ} \mathrm{F}\right)$ por encima de la temperatura ambiente. La clasificación F o $\mathrm{T}$ indica la duración del ensayo de fuego a que ha sido sometido el sistema. También existe la clasificación L ('L rating'), la cual determina el volumen de aire que escapa a través del sistema cortafuegos, tanto a temperatura ambiente como a $204{ }^{\circ} \mathrm{C}\left(400{ }^{\circ} \mathrm{F}\right)$. El propósito de la clasificación L es ayudar a determinar la efectividad de los sellos al restringir el movimiento del humo.

Algunos de estos sistemas de sellos cortafuego permiten poner o quitar fácilmente los elementos que traspasan la pared, facilitando el cambio de los tubos, tuberías o cables. Sin embargo, puede que la restauración de la integridad del producto cortafuego no se pueda hacer en las mismas condiciones que el original. Si hay que hacer cambios que exijan quitar y recolocar el sistema cortafuego, se debe hacer con el máximo cuidado para que: la nueva instalación del cortafuegos se haga de manera aprobada; sólo se instale el número de elementos penetrantes permitido $\mathrm{y}$; se dejen las distancias aprobadas entre los elementos penetrantes y los laterales de los orificios.

\section{TRATAMIENTOS IGNIFUGANTES}

El término ignifugante se emplea en relación con productos químicos, pinturas y recubrimientos utilizados para tratar materiales de construcción combustibles, así como para dichos materiales, una vez protegidos. La ASTM define un material ignífugo como aquel que proporciona índices de inflamabilidad o propagación de llamas comparativamente bajos. El índice nominal de propagación de llama se expresa en números o clasificaciones obtenidas de acuerdo con las especificaciones de la NFPA 255. Los términos "retardador de llamas" y "resistente a las llamas" pueden emplearse más o menos indistintamente para designar materiales de decoración que, debido al tratamiento químico a que se les somete o a sus propiedades inherentes, no se inflaman fácilmente ni propagan las llamas cuando están expuestos a fuegos pequeños o moderados. Para designar productos químicos, pinturas y recubrimientos empleados para el tratamiento de elementos decorativos o de construcción (entre los que se incluyen telas, árboles de navidad, cableado eléctrico, etc.) es preferible emplear el término "retardador de llamas".

Cuadro 1. Sistema de numeración UL para sellos cortafuego

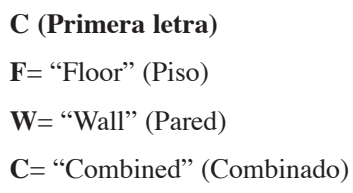

\author{
AJ (Segundas letras) \\ 2165 (Números de serie) \\ 0000 Sin elemento penetrante \\ 1000 Tuberías metálicas \\ 2000 Tuberías no metálicas \\ 3000 Cables eléctricos \\ 4000 Canastas de cables \\ 5000 Tubería aislada \\ 6000 Ductos eléctricos \\ 7000 Ductos mecánicos \\ 8000 Penetraciones múltiples
}


A continuación se establecen tres mecanismos por los que ciertos productos químicos ignifugantes pueden retardar la combustión de los materiales.

\subsection{Por sus características térmicas}

Los productos ignífugos reducen de tres maneras distintas la acumulación de calor en los materiales tratados:

Aumentan la conductividad térmica y por tanto, la disipación del calor de combustión.

Absorben más calor, lo cual reduce la cantidad del mismo disponible para la pirólisis.

Proporcionan aislamiento térmico, lo cual disminuye la transmisión de calor al material. El aislamiento térmico es el mecanismo más eficaz.

\subsection{Por recubrimiento}

Algunos ignifugantes se funden o derriten a temperaturas relativamente bajas y forman un recubrimiento aislante sobre las fibras del material tratado, actuando por medio de la exclusión del oxígeno y la inhibición del escape de los gases combustibles. Otros muestran una acción burbujeante espumante, formando una barrera que las aísla. Las pinturas intumescentes son un buen ejemplo de este mecanismo.

\subsection{Por emisión de gases}

Algunos productos químicos ignifugantes reaccionan bajo los efectos del calor, emitiendo gases como vapor de agua, amoníaco y anhídrido carbónico. De esta forma, se diluyen los gases combustibles hasta que su mezcla con el aire resulta insuficiente para la combustión. Otros ignifugantes actúan como catalizadores de las reacciones en cadena de radicales que se producen en la fase gaseosa (combustión con llama). Los ignifugantes con halógenos experimentan este tipo de proceso y cuando se combinan con antimonio, tienden a sofocar las llamas.

\section{MÉTODOS DE TRATAMIENTO IGNIFUGANTE}

Existen cuatro métodos básicos de tratamientos para que los materiales sean ignífugos: cambios químicos (sustituciones y mezclas); impregnación (saturación y absorción); impregnación bajo presión y recubrimiento.

Los métodos de cambios químicos y de impregnación a presión están generalmente limitados a los procesos de fabricación industriales y no pueden ser empleados sobre el terreno. Los de impregnación y recubrimiento, por el contrario, sí son aptos para su aplicación fuera de la fábrica. Generalmente son preferibles los métodos industriales, ya que proporcionan mayor uniformidad, permanencia y fiabilidad.

\subsection{Transformación química}

Los cambios químicos originados por los ignifugantes son efectivos fundamentalmente en los plásticos y en las fibras sintéticas, ya que pueden elegirse diferentes elementos y compuestos que modificarán las características de combustión del producto final. Puesto que los elementos ignifugantes forman parte integral de la composición química, estos materiales se denominan "inherentemente" ignífugos.

\subsection{Impregnación}

Se llama impregnación a un método para tratamiento de materiales absorbentes. Los productos ignifugantes se disuelven o dispersan en un disolvente, generalmente agua, y el material que hay que tratar se empapa totalmente o se satura con la solución, que también puede aplicarse en forma pulverizada o por la inmersión del material. Cuando hay que tratar grandes volúmenes de hilados, se elimina el exceso de solución haciendo pasar el material saturado a través de rodillos, donde se exprime antes de secarse.

El proceso de impregnación con sales hidrosolubles resulta en la deposición de pequeños cristales 
salinos en la superficie de las fibras. El proceso es puramente físico, sin que se produzca ninguna reacción química. Para aplicar tratamientos más duraderos se forman depósitos insolubles por reacción química u otro medio.

Ciertos productos con base en celulosa, como el papel, placas acústicas y tableros de fibras, durante su proceso de manufactura pasan por una etapa de pulpa húmeda. Es posible añadir productos químicos ignifugantes a esta pulpa húmeda, lo que resulta en una distribución más uniforme en la totalidad de la masa del producto terminado. Esta práctica es predominante en la fabricación de muchos tipos de papeles tratados y actualmente es empleada por muchos productores de materiales de construcción. Las placas acústicas de fibra de madera y las láminas para la construcción tratadas con este método, tienen un índice de propagación de la llama inferior a 25 , de acuerdo con las especificaciones ASTM E-84/ NFPA 255.

\subsection{Impregnación a presión}

El método de impregnación a presión se emplea en tratamientos de ignifugación para materiales no absorbentes relativamente densos, como la madera. Este proceso consiste en sustituir el aire que existe en el interior de la madera con una solución química ignifugante(el productoquímico se deposita a medida que la solución se seca). Usualmente, la solución se introduce en la madera por los métodos de vacío que se emplean en la industria de conservación de las maderas. Comparado con las técnicas simples de impregnación, el método de presión proporciona una mayor penetración y retención química.

\subsection{Recubrimientos}

Hay diversos tipos de recubrimientos ignifugantes, útiles para el tratamiento de muchos materiales. Pueden aplicarse en cualquiera de las etapas de la manufactura del producto e inhibir activamente la propagación de las llamas, hasta cierto punto, o presentar una superficie incombustible sobre la cual las llamas no pueden propagarse. Se emplean predominantemente para el tratamiento de materiales no absorbentes de la construcción que no pueden ser tratados por ningún otro método, para protección de cableado, árboles de navidad y otros materiales decorativos o constructivos.

Existen varios tipos de recubrimientos ignifugantes, entre los que están:

- Pinturas intumescentes: estas pinturas se expanden ante la acción del calor cambiando de un recubrimiento delgado, tipo pintura, a una costra gruesa e hinchada parecida al malvavisco tostado. Esta costra produce alguno o todos de los siguientes efectos: aísla el combustible del calor; aísla combustible del oxígeno; produce gases diluyentes y reduce los gases inflamables. Mantiene su eficacia hasta que se resquebraja por alta temperatura o por el calor sostenido.

- Masillas: se aplican con llana o espátulas para formar un grueso recubrimiento sobre la superficie del material combustible; su consistencia, una vez aplicado, varía desde una superficie dura parecida a la cerámica, a una superficie blanda. Resiste cantidades importantes de calor e inhibe la propagación de la llama, debido a la membrana incombustible que forma.

- Pinturas que producen gases: estos recubrimientos, cuando se calientan, emiten gases incombustibles que diluyen el oxígeno en las cercanías de la superficie protegida, impidiendo que se concentre en cantidad suficiente para mantener la combustión.

- Recubrimientos cementosos y de fibras minerales: generalmente se utilizan sobre el acero estructural para protegerlo de las temperaturas elevadas del incendio.

\section{NOTAS FINALES}

La eficacia de un recubrimiento depende de las propiedades químicas y físicas del material 
sobre el que se aplica, de la efectividad del propio recubrimiento en relación con el tipo de material y de la habilidad de quien realiza la operación.

Los recubrimientos ignifugantes pueden aplicarse con brocha, rodillo o pulverizados. Con las pinturas normales, el objetivo usual es cubrir la mayor cantidad posible de superficie con la menor cantidad de material. El enfoque es distinto cuando se trata de aplicar recubrimientos ignifugantes. Aquí, el objetivo es proporcionar la cantidad de recubrimiento por unidad de superficie que sea necesaria para asegurar un grado adecuado de protección. El espesor de película de las pinturas intumescentes es un factor decisivo en las prestaciones de dichos recubrimientos.

Entre los muchos recubrimientos ignifugantes patentados, varios tienen certificación extendida por laboratorios acreditados. Debe desconfiarse de cualquier pintura llamada "ignifugante" o "a prueba de fuego" que no tenga la certificación, extendida por un laboratorio de ensayos reconocido, sobre de sus características de propagación de la llama.

Los sistemas de sellado contra fuego pueden estar constituidos por uno o por varios productos que deberán colocarse en la forma como lo indique el fabricante y hayan sido probados para obtener la protección establecida en la clasificación.

\section{REFERENCIAS BIBLIOGRÁFICAS}

ASTM. (1983). ASTM E-84, Standard test method for surface burning characteristics of building materials. 1983 Annual Book of ASTM Standards, Vol 04.07.

ASTM. (1983). ASTM E-119, Standard test methods for fire tests of building construction and materials. 1983 Annual Book of ASTM Standards, Vol 04.07.

ASTM. (1983). ASTM E-814, Standard test method for fire tests of through penetration firestops. 1983 Annual Book of ASTM Standards, Vol 04.07 .
Drysdale, D. (1998). Fire dynamics. Second Edition, Wiley, USA.

NFPA. (1999). NFPA 101, Code for safety to life from fire in buildings and structures. Spring Meeting Edition.

NFPA. (1999). NFPA 220, Standard on types of building construction. Spring Meeting Edition.

NFPA. (1999). NFPA 221, Standard for fire walls and fire barrier walls. Spring Meeting Edition.

NFPA. (1999). NFPA251, Standardmethods of tests of fire endurance of building construction and materials. Spring Meeting Edition.

NFPA. (1999). NFPA 255, standard method of test of surface burning characteristics of building materials. Spring Meeting Edition.

NFPA. (1999). NFPA 850, Recommended practice for fire protection for electric generating plants and high voltage direct current converter stations. Spring Meeting Edition.

NFPA. (1999). NFPA 851, recommended practice for fire protection for hydroelectric generating plants. Spring Meeting Edition.

NFPA. (1999). NFPA 921, Guide for fire and explosion investigations. Spring Meeting Edition.

\section{SOBRE EL AUTOR}

\section{Jhymer Rojas Vásquez}

Profesor de la Escuela de Ingeniería Mecánica, Universidad de Costa Rica.

Máster en Ingeniería Mecánica, Univesidad de

Costa Rica

Teléfonos:(506) 207-4548

Facsímil:207-5610

Correo electrónico: jhymer@ fing.ucr.ac.cr 
